\title{
ON THE ACCURACY OF SOLAR MODELLING
}

\author{
S. TURCK- CHIÈZE \\ Service d'Astrophysique \\ C.E.N. Saclay \\ 91191 Gif sur Yvette \\ FRANCE
}

\begin{abstract}
The confrontation between theoretical predictions and observations requires an estimate of the uncertainties of these predictions. Recent results on nuclear reaction rates and photospheric abundances are analysed, in a classical model framework. The role of opacities in the determination of the helium content, the neutrino fluxes prediction and the adiabatic sound speed is discussed. Comments on extra phenomena as mass loss, turbulent mixing and WIMPS are also presented, in the light of very recent seismological results.
\end{abstract}

\section{Introduction}

The estimate of the accuracy of classical solar modelling is fundamental if we consider the solar reference model as a basis for discussing the role of extra phenomena, neglected in the classical theory of stellar evolution, as turbulent mixing, rotation, magnetic field...In this conference, the complexity of the parametrisation of such dynamical effects in stars has been evoked. But, very recently, the impressive interpretation (Christensen-Dalsgaard et al 1985, $1988 \mathrm{~b}$, Gough and Kosovishev 1988) of the $5 \mathrm{mn}$ acoustic mode observations (Grec et al 1983, Duvall et al 1988) in terms of radial distribution of the sound speed or density, added to the multiplication of the neutrino detections (Davis et al 1968, Koshiba 1988, Kirsten 1986, Barabanov et al 1985) push us to reemphasize the idea that the Sun is a privileged place to quantify the relative importance of these phenomena. In paper 1 (Turck-Chièze et al 1988), we have discussed the characteristics of an updated reference model and compared different published models. Most of the noticed differences arise from different choices of the input parameters (nuclear reaction rates, equation of state, initial abundances and opacities). So I shall comment on the role of these ingredients and reevaluate the resulting uncertainties of solar modelling. The second part will be devoted to the constraints on the extra phenomena set by the radial distribution of the sound speed deduced from observations. As examples, I shall discuss the effect of turbulent mixing, mass loss and WIMPS.

\section{Present status}

\subsection{THE ROLE OF THE INGREDIENTS}

Figure 1 shows the radial behaviour of two important quantities influential on neutrinos production and seismology: temperature and mean molecular weight, and the respective role of the ingredients versus depth. In the outer $3 \%$ of the solar radius, the mean molecular weight increases rapidly, all the elements are partially ionised, or even neutral, molecules 
appear, so a refined equation of state (Däppen and Lebreton 1989, Christensen-Dalsgaard et al 1988a) is a necessity together with detailed opacity calculations including molecules and non adiabatic estimate of the sound speed. Convection dominates the outer $27 \%$ of the Sun, so opacity do not play a dominant role there (the temperature gradient is taken as adiabatic), and physical understanding of the dynamical effect of convection is crucial to go beyond the limitations of the reference model regarding the surface abundances of lithium and boron (Cayrel et al 1984) and the extention of the convective zone (Vorontsov 1988, Shibahashi and Sekii 1988). This region is certainly the most difficult to simulate today but is very crucial for evolved stars. In the case of the Sun, the age and the radius are precisely known, so the mixing length parameter $\alpha$ is determined along with the approximate size of the convective zone and we can partly disconnect this external region from the internal one.

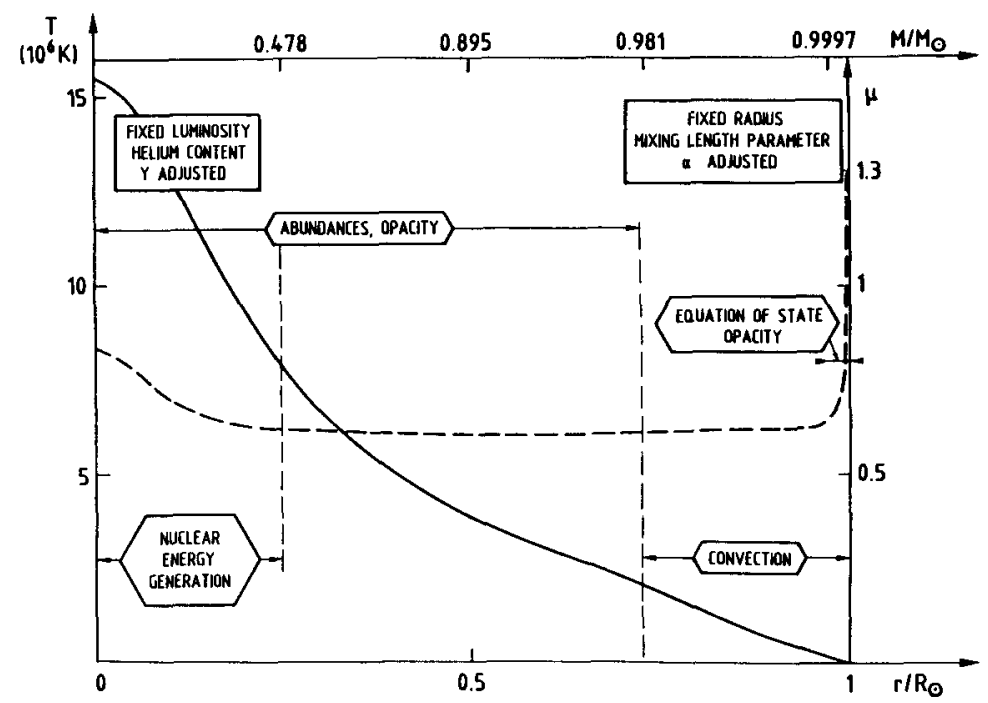

Figure 1: Radial distribution of the temperature $(T \rightarrow$ and the mean molecular weight $(\mu-)$ of our reference model (paper1) and sites of privileged importance of the ingredients.

Thus I shall concentrate, in this paper, on the inner 70-75\% radiative part of the Sun, which has been thoroughly studied this last 20 years. Here, the equation of state plays a minor role: the gas is nearly perfect, hydrogen and helium are completely ionised and the effect of the other elements is smaller than $1 \%$. The behaviour of the "intermediate" region between nuclear core and convective zone is dominated by the opacity coefficients. The central part is influenced by the nuclear reactions rates and the abundances through the opacity coefficients. The initial helium content, unobservable in the photosphere, is adjusted to fit the present luminosity. So, abundances, opacities and nuclear reaction rates are tightly correlated in this inner $25 \%$ of the solar radius.

\subsection{THE REFERENCE MODEL}

An updated reference model offers the opportunity to choose the direction on which effort must be stressed in the classical framework. The model of Turck-Chièze et al (1988) based upon a Paczynsky code includes new nuclear data: neutron lifetime determined by Bopp et al (1986), the recent value of Krauss (1987) for the rate of the reaction ${ }^{3} \mathrm{He}\left({ }^{3} \mathrm{He}, \mathrm{p}\right){ }^{4} \mathrm{He}$ and the 
value recommended by Filippone (1986) for ${ }^{3} \mathrm{He}(\alpha, \gamma){ }^{7} \mathrm{Be}$. The opacities are derived from the Los Alamos library (Huebner et al 1977) using the relative abundances of Ross and Aller (1976), the equation of state includes the Coulomb interaction in the Debye-Huckel approximation. The initial abundances are taken from Aller (1986) and the adopted ratio of the metal abundance to hydrogen (by mass) $\mathrm{Z} / \mathrm{X}$ is $0.028 \pm 0.03$ according to Aller (1986) and Meyer (1987). The main results are the following:

$$
\begin{array}{lllr}
\text { initial helium content } & Y=0.273 \pm 0.012 & \mathbf{r}_{\mathrm{BCZ}}=0.73 \\
\mathrm{~T}_{\mathrm{C}}=15.510^{6} \mathrm{~K} & \rho_{\mathrm{C}}=150 \mathrm{~g} / \mathrm{cm}^{3} & \mathrm{~T}_{\mathrm{BCZ}}=2.02 \\
\text { neutrino capture rates (SNU) } \quad \mathrm{71}_{\mathrm{Ga}: 124 \pm 5} & { }^{37} \mathrm{Cl}: 5.7 \pm 1.3
\end{array}
$$

The chlorine prediction is about three times greater than the experimental average (19681986) of the Davis experiment but nearly consistent with the result of 1987 (4.2 \pm 0.7 SNU) (Davis 1989).

The acoustic frequencies of this model have been computed (Turck-Chièze, Däppen and Cassé 1988). The absolute low degree p-modes are systematically underestimated by about $10 \mu \mathrm{Hz}$ compared to the observations (Grec, Fossat and Pomerantz 1983, Duvall et al 1988). The calculated $\Delta v_{20}$ (see previous references for definition) is about $1 \mu \mathrm{Hz}$ greater than the observed one. Pressure p-modes are sensitive to the whole structure of the Sun and particularly to the outer which is estimated with less care than the inner part. Since the accuracy of the data and of the inversion technique improves so rapidly, I prefer to rely on the adiabatic sound speed which allows to explore different depths of the Sun. Figure 2 illustrates the difference between our reference model and the sound speed derived from the observations by Christensen Dalsgaard, Gough and Thompson (1988). The relative difference in the square of the adiabatic sound speed is lower than $2 \%$ in the radiative region. This implies a variation of the ratio $\mathrm{T} / \mu$ not greater than $2 \%$, with possible greater effect on $\mathrm{T}$ and $\mu$ individually. Assuming a polytropic behaviour $(P=\rho \gamma)$, one can deduce the sign of the variation of $\rho$ and its amplitude assuming a nearly perfect gas $\left(c^{2}=5 / 3 \mathrm{P} / \rho\right)$. We notice a polytropic exponent of 1.2 in our models, suggesting density variation $<10 \%$.

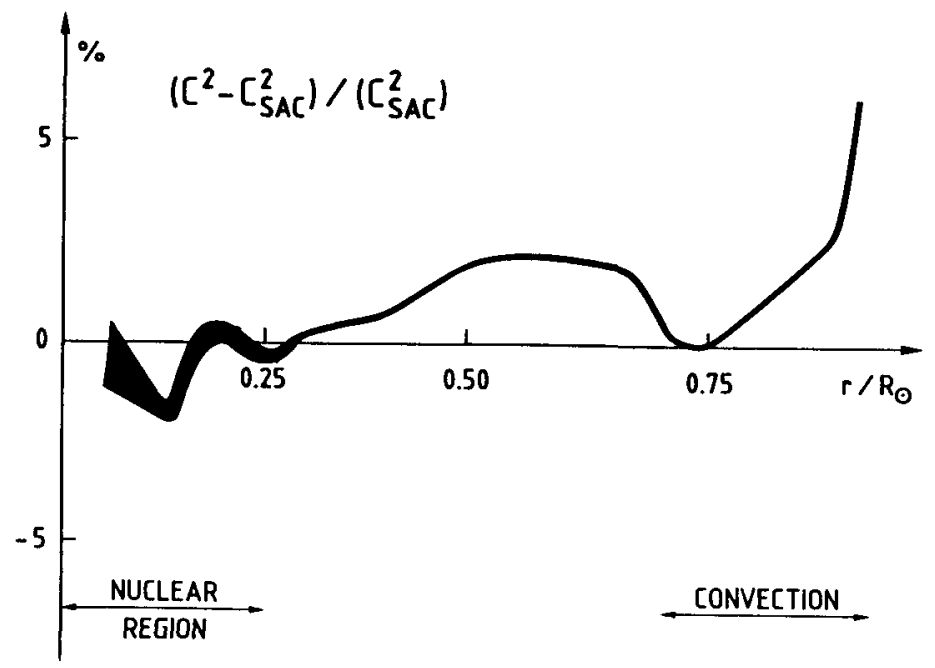

Figure 2: Comparison of the square of the adiabatic sound speed deduced from the observations by Christensen Dalsgaard et al (1988b) and that of paper 1. 


\subsection{PRESENT ACCURACY OF THE REFERENCE MODEL}

In paper 1 , the different contributions to the uncertainty on the predicted neutrino capture rates and helium content are discussed. The corresponding logarithmic derivatives of the sound speed will be published elsewhere (Turck-Chièze and Däppen 1989). Except for the gallium prediction, where the accuracy is quite good, we have pointed out two main sources of unaccuracy: the ratio $\mathrm{Z} / \mathrm{X}$ and the central opacities. We stressed also the important role of the ${ }^{7} \mathrm{Be}(\mathrm{p}, \gamma)$ reaction rate for the chlorine prediction which is, in fact, the key reaction for chlorine (70\% of the neutrinos produced) and water (100\%) detectors (Turck-Chièze 1988).

\subsubsection{The nuclear reaction rates and the case of ${ }^{7} B e(p, y){ }^{8} B$.}

All the reactions of the p-p chain and the CNO cycle have been remeasured recently, most of the cross sections are determined at a level of 5\%. (Rolf 1989). Each individual experiment has more limited accuracy but one usually uses the average value of all the different experiments and the corresponding error bar. Moreover they are most generally performed at higher energies than the astrophysical range (typically $20 \mathrm{keV}$ for central solar conditions), so a theoretical extrapolation is necessary. As an illustration I discuss the case of ${ }^{7} \mathrm{Be}(\mathrm{p}, \gamma)$. The experimental situation is summarised in Parker (1986) and recalled in table 1 . The mean value is recommended by Filippone (1983). But as usual, the compilation is presented in terms of the astrophysical factor $S_{17}(0)$ derived with, in each case, the crude theoretical extrapolation of Tombrello (1965). This theoretical calculation has been revised by Barker $(1980,1983)$ and more recently by Kajino (1988) (figure 3 ). These two calculations, including not only s-state (as in the case of Tombrello) but also d-state, are in agreement together and lead to a reduction of $S_{17}(0)$ of $15 \%$. We adopt the mean experimental value recommended by Filippone (1986) but the most recent theoretical results. $S_{17}(0)$ is reduced to $0.021 \mathrm{keV}-b$. The chlorine neutrino capture rate is consequently reduced by $13 \%$. This choice constitutes one of the differences with Bahcall and Ulrich work (1988). We note that the experimental results are very scattered and remeasurement on the whole range $100 \mathrm{keV}-4 \mathrm{MeV}$ would be useful. It is the reason why we adopt an error bar of $15 \%$.

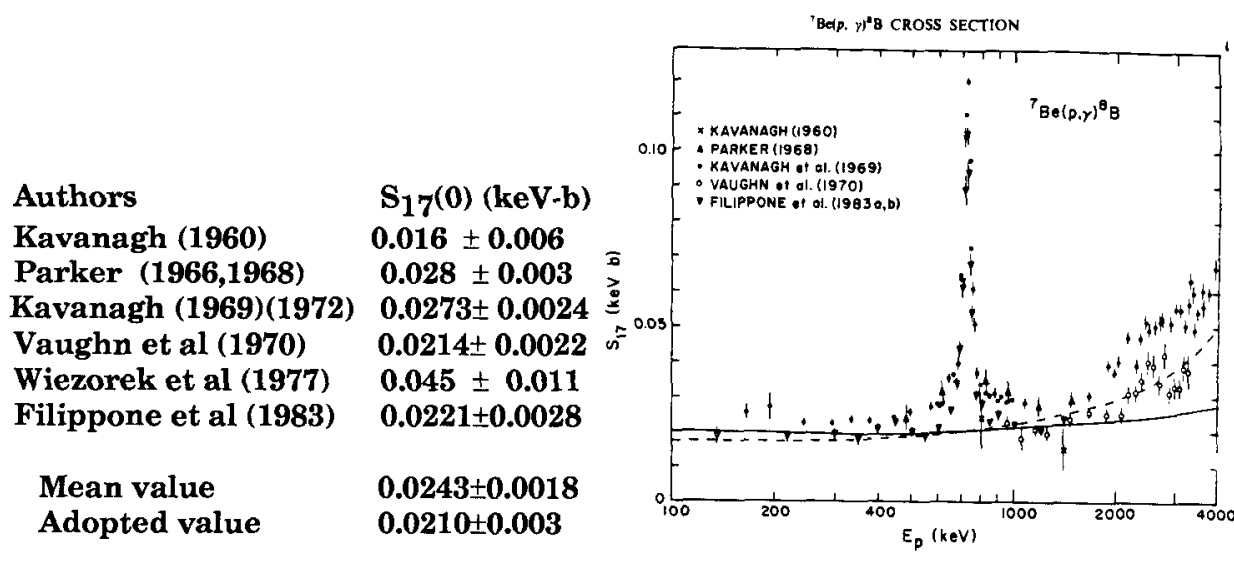

Table 1

Figure 3.Experimental measurements and theoretical extrapolation of the astrophysical factor with Tombrello (1965) (-) and Barker (1980) (- -) calculations. 
Very recently, Assenbaum et al (1987) have noticed that electron screening affects the measured cross sections at low energy, and increases exponentially, the experimental value. Since, the nuclei are mainly bare in the central Sun, this effect leads to an overestimate of $\mathrm{S}(0)$. It could concern ${ }^{3} \mathrm{He}\left({ }^{3} \mathrm{He}, 2 \mathrm{p}\right)^{4} \mathrm{He}(6 \%)$ and ${ }^{3} \mathrm{He}(\alpha, \gamma){ }^{7} \mathrm{Be}$ and has never been taken into account in the estimation of the uncertainties on the neutrino capture rates.

\subsubsection{Role of abundances and effect of opacity.}

The initial composition of the Sun is assumed to be similar to the present observable photospheric one. During hydrogen burning, the p-p chain dominates the production of energy and the CNO cycle contribution is of only $1.75 \%$. So only elements up to oxygen participate to the nuclear reactions. On the other hand, the opacity, which regulates the energy flux in the radiative region of the star and determines its structure, is largely influenced by the heavy elements (up to iron), they contribute to $40 \%$ of the total opacity in the center and to $90 \%$ in what we call the "intermediate" radiative region. In this context, the determination of the solar composition is influential on the central structure of the Sun. In our computation, we fix $Z / X$ according to the observations as Bahcall and Ulrich (1988). As a consequence, the initial helium content and the metallicity cannot be chosen independently: if the helium content increases, the metallicity must decrease, so that $\mathrm{Z} / \mathrm{X}$ remains equal to the observed value. This constraint requires to interpolate between two opacity tables $\kappa=\kappa(T, p, X, Z)$. Consequently, the transformation of carbon and oxygen into nitrogen during hydrogen burning is taken into account, at the first order, as an enhancement of the metallicity by $3 \%$. This method is justified by the similarity of the opacity behaviour of C,N,O for central solar conditions. This CNO evolution is treated differently in Bahcall and Ulrich paper(1988). In the poster entitled "The influence of metallicity on the opacity coefficients in the solar modelling" (Courtaud et al 1989), we discuss two sources of uncertainties on the opacity coefficients : the relative composition of the Sun at birth and the accuracy of the calculations. In the nuclear central part, the accuracy of the calculations is good $(5 \%)$, the possible variations of the opacity could arise from the determination of the abundances. We observe variations of the central structure using Ross and Aller (1976) composition in the opacity calculation with $\mathrm{Z} / \mathrm{X}=0.028$ or Anders and Grevesse new compilation (1989) with $\mathrm{Z} / \mathrm{X}=0.0273$. We notice also that the unexplained discrepancy between photospheric $\left(\mathrm{Fe} / \mathrm{X}=4.68 \pm 0.3310^{-5}\right)$ and meteoritic $(\mathrm{Fe} / \mathrm{X}=$ $3.24 \pm 0.07510^{-5}$ ) iron values could lead to opacity variations up to $13 \%$ and a related reduction of the chlorine neutrino capture rate of 1 SNU. In the intermediate region,the accuracy of the opacity calculations is at a level of $20 \%$ and could increase the temperature of the bottom of the convective zone by $10 \%$, with few effect on the central structure.

Electron collective effects must be taken into account in the calculation of the central solar opacities. It is not clear if this process is included in the library of Los Alamos 1977. We have always considered that it was not the case, following the discussion of Bahcall et al (1982) and Huebner (1986). In our computation, this correction is added according to the expression : $\kappa=\kappa-0.07(1+\mathrm{X}) \mathrm{cm}^{2} \mathrm{~g}^{-1}$ (Bahcall et al 1982). This point must be clarified in the near future to avoid counting this effect twice or not at all. A suppression of this additive correction increases the chlorine neutrino capture rate by $0.6 \mathrm{SNU}$ and the helium content by 0.006 . Boercker (1987) independently, has reconsidered this correction to the Thomson scattering and concludes that the different estimates lead to an error on the total opacity of $\pm 2.5 \%$ at the center of the Sun.

In summary, the principal ingredients are determined at a level of 5\%-10\%accuracy. A list of reasons has been evoked to justify small variations of the central temperature and perhaps $30 \%$ on the chlorine neutrino flux. But it seems difficult to find classical effects which reduce this flux by a factor 3 or 4 . So room is left for extra phenomena. 


\section{Effect of other phenomena}

As a first approach, we have considered two additional physical effects (Turck-Chièze and Däppen (1989):-an hypothetical mass loss in the early life of the Sun

- the influence of WIMPS in the inner part of the star (see poster untitled

"WIMPS and solar evolution codes" by Giraud-Héraud et al ).

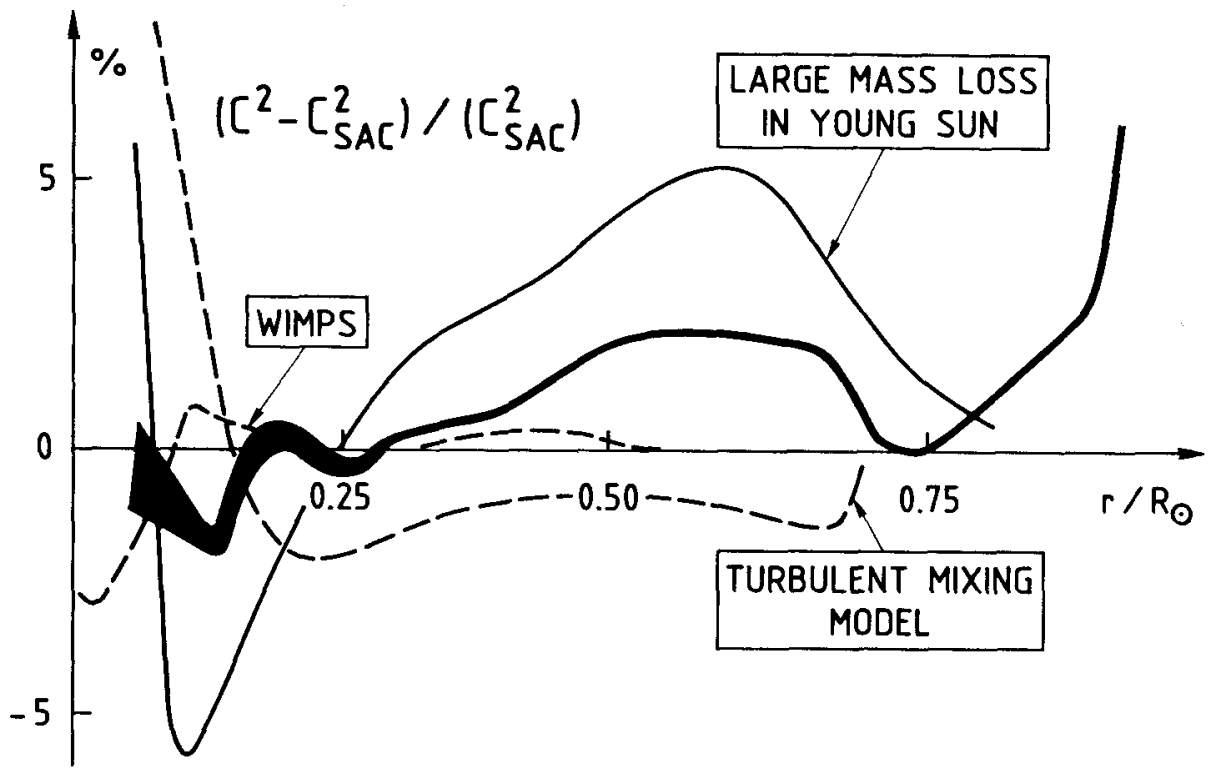

Figure 4 : Radial distribution of the squared sound speed for models including extra phenomena.

Figure 4 shows that, if one believes the extraction of the sound speed from the helioseismological data and the corresponding error bar, all the models presented here are strongly constrained or excluded:

- The present Sun (and especially its global oscillations) is unconsistent with a total initial mass loss greater than $0.2 \mathrm{M}_{\odot}$ (Turck-Chièze, Däppen and Cassé 1988), the neutrino predictions are not reduced. In the case of initial $2 \mathrm{M}_{\odot}$ (fig. 4), an initial convective core could persist at the present day (if overshooting is included), which produces a modification of the structure, at low radius, of too large amplitude but suggestive of the one deduced from the observation.

- Even if for some special conditions of mass and interaction cross section, the introduction of WIMPS appreciably reduces the neutrino capture rate as expected, the sound speed profile of the model leads to a marked disagreement with observation, at low radii. This was not predictable by looking only to the integrated value of $\Delta v_{20}$.

- Concerning turbulent mixing, the difficulty comes from the determination of the temporal and spatial evolution of this phenomenon. From the examination of the work of Schatzman et al (1981) it appears now clearly that the proposed effect is certainly surestimated, due to an overestimate of the chlorine capture rate prediction. Nevertheless, if the consequent effect on the sound speed is excessive, the inner profile is interestingly not far from that deduced from the observations. 


\section{Comments}

In the inner radiative region of the Sun, most of the input parameters are now estimated within $10 \%$ accuracy or even less, possible new sources of unaccuracy are critically examined: electron screening effect in cross section measurements at low energy, electron collective effect in opacity calculations, role of partially ionised heavy elements in opacity calculations. In the classical framework, the internal structure of the Sun seems more and more established and only small variations of temperature and density are possible. The 27$30 \%$ external part is more questionable due to dynamical effects as convection; detailed equation of state and nonadiabatic estimate of sound speed are a necessity for the $3 \%$ outer.

We consider that the predicted ${ }^{8} \mathrm{~B}$ neutrino flux accuracy is not yet well established and propose different possible sources of uncertainties: the measurement of ${ }^{7} \mathrm{Be}(\mathrm{p}, \gamma)^{8} \mathrm{~B}$ reaction rate $(15 \%)$ and the determination of the central opacities $(20 \%)$ due to evolution of detailed abundances. We emphasize the importance, for this quantity, to solve the discrepancy between photospheric and meteoritic iron abundance.

The confrontation of the classical theory of the Sun evolution with the observations shows that the thermodynamical quantities of an updated reference model are representative of the real Sun at a level of $10 \%$ accuracy in the radiative region: neutrino fluxes detection mainly constrains the temperature of the central region $(\mathrm{r}<0.15 \mathrm{RO})$, helioseismology constrains the inner density in the same region. Following Kosovishev (1989), and our recent results on neutrino fluxes, a certain compatibility seems to exist between seismological interpretation and present chlorine detection. Is temporal evolution of these two kinds of information an help to interpret a larger reduction of neutrino flux as was observed before?. The study of dynamical effects is now largely encouraged by the seismological constraints in the central part. The hypothesis of WIMPS seems more questionable but central limited mixing and very weak mass loss cannot be excluded.

\section{Acknowledgements}

This work was initiated four years ago by M. Cassé and is the fruit of a large collaboration. The stellar evolution code was provided by J. P. De Greve, B. de Loore and C. Doom, the Los Alamos opacities by A. Noels and R. Papy, the nucleosynthesis was performed by $N$. Prantzos, the seismological interpretation was done with W. Däppen. More recently, a collaboration was engaged with D. Courtaud, G. Damamme, E. Genot and M. Vuillemin to critically discuss the opacity determination, and with A. Bouquet, Y. Giraud-Héraud, J. Kaplan, F. Martin, C. Tao for the study of the effect of WIMPS. Basic results were obtained with S. Cahen and C. Doom. I am very grateful to all of them.

\section{References}

Aller,L. H.,1986, in Spectroscopy of Astrophysical Plasmas, ed A. Dalgarno and D. Layzer (Cambridge: Cambridge University Press) p 89.

Anders, E. and Grevesse, N., 1989, Geochim. Cosmochim. Acta, 53, 197.

Assenbaum, H.J., Langanke, K., and Rolfs, C. 1987, Z. Phys. A-Atomic Nuclei, 327, 461.

Bahcall,J.N., Huebner,W.F., Lubow,S.H., Parker,P.D., and Ulrich,R.K. 1982, Rev. Mod. Phys., 54, 767.

Bahcall, J. N., and Ulrich, R. K. 1988, Rev. Mod. Phys., 60, 297.

Barabanov, I. R., et al 1985, in Solar Neutrinos and Neutrino Astronomy, ed. M. L. Cherry, K. Lande, and W. A. Fowler (New York:AIP), p. 175.

Barker, F. C. 1980, Aust. J. Phys., 33, 177. 
Barker, F. C. 1983, Phys. Rev. C, 28, 1407.

Boercker, D. B. 1987, Ap. J. (Letters), 316, L95.

Bopp, P., Dubbers, D., Hornig, L., Klemst, E., Last, A., and Schultze, H. 1986, Phys. Rev. Lett., 56, 919.

Cayrel, R., Cayrel De Strobel, G., Campbell, B., Däppen, W. 1984, Ap. J., 283, 205.

Christensen-Dalsgaard, J., Duvall, T. L., Jr, Gough, D. O., Harvey, J.W., Rhodes, E. J., Jr, 1985, Nature, , 315, 378.

Christensen-Dalsgaard, J., Däppen, W., Lebreton, Y. 1988a, Nature, 336, 634.

Christensen-Dalsgaard, J., Gough, D. O., Thompson, M. J. 1988b, in Seismology of the Sun and Sunlike stars, ESA SP-286, 493.

Courtaud,D., Damamme,G., Genot,E., Vuillemin,M., Turck-Chièze,S. 1989, This conference.

Däppen, W. and Lebreton, Y. 1989, Astr. Ap., in preparation. and references therein.

Davis, R., Jr., Harmer, D. S., and Hoffman, K. C. 1968, Phys. Rev. Letters, 20, 1205.

Davis, R. 1989, These proceedings.

Duvall, T.L. , Harvey, J. W., Libbrecht, K.G., Popp, B.D., and Pomerantz, M. A. 1988, Ap. J., 324,1171.

Filippone, B. W., Elwyn, A. J., Davids, C. N., Koetke, D. D. 1983, Phys. Rev. Lett., 50, 412. and Phys. Rev. C, 28, 2222.

Filippone, B. W. 1986, Ann. Rev. Nucl. Part. Sci.,36, 717.

Giraud-Héraud,Y., Kaplan, J., Martin, F., Tao, C., Turck-Chièze, S., 1989, Solar Phys.,This conference.

Grec, G., Fossat, E. and Pomerantz, M. A., 1983, Solar Phys., 82, 55.

Gough,D.O., and Kosovishev,A.G. 1988, in Seismology of the Sun and Sun-like stars, ESA SP-286, 195.

Huebner, W. F., 1986, in Physics of the Sun, Vol 1, ed. P. A. Sturrock (Dordrecht: Reidel), p. 33.

Huebner, W. F., Merts, A.L., Magee, N. H., Jr., and Argo, M.F. 1977, Los Alamos Sci. Lab. Rept., LA6760-M.

Kajino, T., Bertsch, G. F., and Barker, F. C. 1988, in Clustering Aspects in Nuclear and Subnuclear Systems, to appear in Suppl. J. Phys. Soc. Japan

Kirsten, T. 1986, in 86 Massive Neutrinos in Astrophysics and in Particle Physics, ed. O. Fackler and J. Tran Thanh Van (Ed. Frontières, Gif sur Yvette) p119.

Koshiba, M. T. 1988, in 5th Force and Neutrinos Physics, ed. O. Fackler and J. Tran Thanh Van (Ed. Frontières, Gif sur Yvette) p 215.

Kosovishev, A.G. 1989, These proceedings.

Krauss, A., Becker, H.W., Trautvetter, H.P., and Rolfs, A. 1987, Nucl. Phys. A, 467, 273.

Meyer, J. P. 1987, in Origin and Distribution of the Elements, ed. G. J. Matthews (Singapore: World Scientific), p 337.

Parker, P. D. McD. 1986, in Physics of the Sun, Vol 1, ed. P. A. Sturrock (Dordrecht: Reidel), p. 17.

Rolf, K. 1989, These proceedings.

Ross, J.E., and Aller, L. H. 1976, Science, 191, 1223.

Schatzman, E., Maeder, A. 1981, Astr. Ap., 96, 1.

Shibahashi, H., and Sekii, T. 1988, in Seismology of the Sun and Sun-like stars, ESA SP-286, 471.

Tombrello, T. A. 1965, Nucl. Phys., 71, 459.

Turck-Chièze, S. 1988, in 5th Force and Neutrinos Physics, ed. O. Fackler and J. Tran Thanh Van Ed. Frontières, Gif sur Yvette) p 165.

Turck-Chièze, S., Cahen, S., Cassé, M., Doom, C. 1988, Ap. J., 335, 415.

Turck-Chièze, S., Dappën, W. , Cassé, M. 1988, in Seismology of the Sun and Sun-like stars, ESA SP286, 629.

Turck-Chièze, S., Däppen, W., 1989, in preparation.

Vorontsov S. V. 1988 in Seismology of the Sun and Sun-like stars, ESA SP 286, 475. 